\title{
DELP: Dynamic Epistemic Logic for Security Protocols
}

\author{
Ioana Leuștean and Bogdan Macovei \\ Faculty of Mathematics and Computer Science \\ University of Bucharest \\ Bucharest, Romania \\ ioana.leustean@unibuc.ro, bogdan.macovei@unibuc.ro
}

\begin{abstract}
The formal analysis of security protocols is a challenging field, with various approaches being studied nowadays. The famous Burrows-AbadiNeedham Logic was the first logical system aiming to validate security protocols. Combining ideas from previous approaches, in this paper we define a complete system of dynamic epistemic logic for modeling security protocols. Our logic is implemented, and few of its properties are verifyied, using the theorem prover Lean.
\end{abstract}

\section{Introduction}

This paper presents $D E L P$, a dynamic epistemic logic for analysing security protocols. In order to define our logic, we combine the epistemic approach to authentification from [5], the expectation semantics from [8] and the operational semantics for security protocols from [7].

Our main contributions are: (i) the definition of DELP as a sound and complete system with respect to an expectation semantics representing the adversary knowledge; (ii) the implementation of DELP in the theorem prover Lean. Consequently, using Lean: (iii) we defined translations in DELP for a few inference rules of the Burrows-Abadi-Needham $(B A N)$ logic [4] and we proved their soundness, (iv) we defined the Needham-Schroeder authentication protocol as a theory in DELP and we verified a few security claims.

Section 2 presents the Needham-Schroder security protocol and recalls the formal approaches from [5], [8] and [7]. In Section 3 we define the system DELP and we prove its properties. Section 4 contains the Lean implementation of $D E L P$. Few deduction rules of the BAN Logic are defined in DELP and their soundness is proved using the Lean implementation. In Section 5 we study the Needham-Schroeder authentication protocol using DELP and its Lean implementation. The last section contains conclusions and further developments. 


\section{Preliminaries: formal analysis of security pro- tocols}

A security protocol is defined as a set of rules and conventions that determine the exchange of messages between two or more agents in order to implement a security service. The protocol must be unambiguous and must allow the description of several roles, so that an agent can perform a certain role at a certain protocol round. An example of a security protocol, which we will mention and use in this paper, is the Needham-Schroeder protocol.

\subsection{The Needham-Schroeder symmetric key protocol for key exchange}

The protocol specification for three agents is as follows:

$$
\begin{aligned}
& A \rightarrow S: A, B, N_{a} \\
& S \rightarrow A:\left\{N_{a}, B, K_{a b},\left\{K_{a b}, A\right\}_{K_{b s}}\right\}_{K_{a s}} \\
& A \rightarrow B:\left\{K_{a b}, A\right\}_{K_{b s}} \\
& B \rightarrow A:\left\{N_{b}\right\}_{K_{a b}} \\
& A \rightarrow B:\left\{N_{b}-1\right\}_{K_{a b}}
\end{aligned}
$$

A step-by-step description of the protocol is:

1. Alice initiates the connection with the Server, sending who she is, with whom she wants to communicate and a nonce;

2. the Server sends - encrypted with the common key between Alice and Server - the nonce generated by Alice, the identity of Bob and the communication key between Alice and Bob, to which is added a message that only Bob can decrypt (being encrypted with the communication key between Bob and Sserver), which contains the communication key shared by Alice and Bob; in this way, Alice cannot read the message sent by Server to Bob;

3. Alice sends Bob the message that it could not decrypt, received from the Server;

4. Bob decrypts the message, and sends Alice a nonce encrypted with the common key between Alice and Bob;

5. Alice receives Bob's message, decypts it, and resends it, applying a simple function to it - in this case, it decrements it. This step is useful in two situations: it is a first protection on a reply attack and it shows that the agents are still alive in the session.

\subsection{BAN Logic}

We will briefly present the BAN logic, based on [4]. The mathematical system contains the following sets: a set of participating agents in communication protocol sessions - named, generally, using capital letters of the beginning of the 
alphabet $(\mathrm{A}, \mathrm{B}, \ldots)$, a set of keys - named, generally, $K_{a, b}$ for the public key between agents A and B, $K_{a}$ for A's public key and $K_{a}^{-1}$ for A's secret key, and a set of messages - named, generally, using capital letters of the end of the alphabet $(\mathrm{X}, \mathrm{Y}, \ldots)$. An encrypted message is denoted by writing $\{X\}_{k}$, meaning that the message $X$ is encrypted with the key $k$.

The specific formulas introduced in BAN logic are the following:

- $P \mid \equiv X$ : the agent $P$ believes the message $X$;

- $P \triangleleft X$ : the agent $P$ sees or receives $X$;

- $P \mid \sim X$ : the agent $P$ once said or sends $X$;

- $P \Rightarrow X$ : the agent $P$ controls $X$ or have jurisdiction over $X$;

- $\#(X): X$ is a nonce;

- $P \stackrel{k}{\leftrightarrow} Q$ : the agents $P$ and $Q$ shares the communication key $k$;

- $\stackrel{k}{\mapsto} P: k$ is $P$ 's public key;

- $\{X\}_{k}: X$ is encrypted with the key $k$;

- $<X>_{Y}: X$ is encrypted with the common secret $Y$.

In the sequel we recall only two deductions rules, we refer to [4] for the full deduction system.

The Message Meaning Rule, formally defined by

$$
\frac{P \mid \equiv Q \stackrel{K}{\leftrightarrow} P \quad P \triangleleft\{X\}_{K}}{P|\equiv Q| \sim X}
$$

can be read as follows: if agent $\mathrm{P}$ belives that he has a communication key $K$ with agent $\mathbf{Q}$, and agent $\mathbf{P}$ receives a message $\mathbf{X}$ encrypted under $K$, then $P$ belives that the encrypted message was sent by Q.

The Jurisdiction rule, formally defined by

$$
\frac{P|\equiv Q \Rightarrow X \quad P| \equiv Q \mid \equiv X}{P \mid \equiv X}
$$

can be read as follows: if agent $P$ belives that agent $Q$ has jurisdiction over a message $\mathrm{X}$ and, furthermore, agent $\mathrm{P}$ belives that $\mathrm{Q}$ belives $\mathrm{X}$, then $\mathrm{P}$ belives $\mathrm{X}$.

\subsection{An approach based on epistemic logic}

In this subsection, we recall the main ideas from [5], and we refer to [9] for a comprehensive presentation of dynamic epistemic logic.

In this paper, there are defined $K$ (the set of communication keys), $N$ (the set of nonces), $T$ (the set of plain texts) and $\Phi$ (the set of formulas). The BNF 
specification of the language is:

$$
\begin{aligned}
\mathbf{s}::= & s \mid x \\
\mathbf{m}::= & t|k| n|i|\left(m_{1}, m_{2}\right)\left|\{m\}_{k}\right| \varphi \\
\varphi::= & p\left|\operatorname{sent}_{i}(s)\right| \operatorname{recv}_{i}(s)\left|\operatorname{extract}_{i}(m)\right| \neg \varphi\left|\varphi_{1} \wedge \varphi_{2}\right| K_{i} \varphi \mid \\
& \bigcirc \varphi|\bigcirc \varphi| \square \varphi|-\varphi| \exists x \varphi|[m]=s| s \sqsubseteq s^{\prime} \mid \operatorname{Pr}_{i}(\varphi) \geq \alpha
\end{aligned}
$$

where $p$ is an atomic formula, $i$ is an arbitrary agent, $m$ is an arbitrary message, $t \in T, k \in K, n \in N, \alpha \in[0,1]$ a probability, $s$ a string, $x$ a variable over strings and $\varphi \in \Phi$.

For semantics, the models are

$$
I=\left(R, \pi, \mathbf{C},\left\{\mu_{C}\right\}_{C \in \mathbf{C}}\right)
$$

where $R$ is a protocol rounds system, $\pi$ is an evaluation function, $\mathbf{C}$ is a partition of $R$, and for every $C \in \mathbf{C}$, the measure $\mu_{C}$ is the distribution probability over rounds in $C$. The inductive interpretation of formulas in this models are:

$$
\begin{aligned}
&(I, r, m) \models p \Longleftrightarrow \pi(r(m))(p) \text { is true } \\
&(I, r, m) \models \neg \varphi \Longleftrightarrow(I, r, m) \not \varphi \models(I, r, m) \models \varphi_{1} \wedge \varphi_{2} \Longleftrightarrow(I, r, m) \models \varphi_{1} \text { and }(I, r, m) \models \varphi_{2} \\
&(I, r, m) \models K_{i} \varphi \Longleftrightarrow \text { for all }\left(r^{\prime}, m^{\prime}\right) \sim_{i}(r, m), \\
&(I, r, m) \models \text { we have }\left(I, r^{\prime}, m^{\prime}\right) \models \varphi \\
&(I, r, m) \models \bigcirc \varphi \Longleftrightarrow(I, r, m+1) \models \varphi \\
&(I, r, m) \models \bigodot \varphi \Longleftrightarrow m=0 \text { or }(I, r, m-1) \models \varphi \\
&(I, r, m) \models \square \varphi \Longleftrightarrow \text { for all } m^{\prime} \geq m,\left(I, r, m^{\prime}\right) \models \varphi \\
&(I, r, m) \models--\varphi \Longleftrightarrow \text { for all } m^{\prime} \leq m,\left(I, r, m^{\prime}\right) \models \varphi \\
&(I, r, m) \models P r_{i}(\varphi) \geq \alpha \Longleftrightarrow \alpha \\
& \mu_{r, m, i}\left(\left\{\left(r^{\prime}, m^{\prime}\right) \mid\left(I, r^{\prime}, m^{\prime}\right) \models \varphi\right\} \cap K_{i}(r, m) \cap(C)(r)\right) \geq \alpha \\
&(I, r, m) \models \exists x \varphi \Longleftrightarrow \text { exists } s \text { string, }(I, r, m) \models \varphi[s / x]
\end{aligned}
$$

\subsection{An approach based on operational semantics}

From [7], the main point of interest is the terms deduction system. In this formal system we have terms (roles, messages, keys and nonces), variables over Var, Fresh and Role sorts, functions symbols (in Func), the protocols specifications and a labeled transition system for the execution of the protocols.

Having $\Gamma$ a knowledge set, the term deduction rules are:

- if $t \in \Gamma$, then $\Gamma \vdash t$;

- $\Gamma \vdash t_{1}$ and $\Gamma \vdash t_{2}$ if and only if $\Gamma \vdash\left(t_{1}, t_{2}\right)$;

- if $\Gamma \vdash t$ and $\Gamma \vdash k$, then $\Gamma \vdash\{t\}_{k}$;

- if $\Gamma \vdash\{t\}_{k}$ and $\Gamma \vdash k^{-1}$, then $\Gamma \vdash t$;

- if $\Gamma \vdash t_{i}, 1 \leq 1 \leq n$, then $\Gamma \vdash f\left(t_{1}, t_{2}, \ldots, t_{n}\right)$, where $f$ is a function symbol of Func, with the arity $n$. 


\subsection{An approach based on expectation models}

In this subsection, we will present the main results of [8], that we will use in the next section to prove the completeness theorem of our system.

In this paper there are introduced two sets, $I$ - the set of agents and $P$ the set of formulas. For interpreting formulas there are used Kripke models, $\mathcal{M}=(S, \sim, V)$, where $S$ is the set of accessible world, $\sim$ is the accessibility relation between worlds and $V$ is the evaluation function, $V: P \rightarrow \mathcal{P}(S)$.

There are an action set $-\Sigma$ - and a langue of observations $-\mathcal{L}_{\text {obs }}$. The BNF grammar of the actions is:

$$
\pi::=\delta|\varepsilon| a|\pi \cdot \pi| \pi+\pi \mid \pi^{*}
$$

where $\delta$ is an empty set of observations, $\varepsilon$ is the empty string and $a \in \Sigma$.

The observations set is denoted by $\mathcal{L}(\pi)$ and is inductively defined as:

$$
\begin{aligned}
& \mathcal{L}(\delta)=\emptyset \\
& \mathcal{L}(\varepsilon)=\{\varepsilon\} \\
& \mathcal{L}(a)=\{a\} \\
& \mathcal{L}\left(\pi \cdot \pi^{\prime}\right)=\left\{w v \mid w \in \mathcal{L}(\pi) \text { and } v \in \mathcal{L}\left(\pi^{\prime}\right)\right\} \\
& \mathcal{L}\left(\pi+\pi^{\prime}\right)=\mathcal{L}(\pi) \cup \mathcal{L}\left(\pi^{\prime}\right) \\
& \mathcal{L}\left(\pi^{*}\right)=\{\varepsilon\} \cup \bigcup_{n>0}(\mathcal{L}(\pi \cdot \ldots \cdot \pi))
\end{aligned}
$$

An epistemic model defined with this observations is an epistemic expectation model $\mathcal{M}=(S, \sim, V, \operatorname{Exp})$, where $\operatorname{Exp}: S \rightarrow \mathcal{L}_{\text {obs }}$ is a function that maps every state from $S$ to an observation $\pi$ for which $\mathcal{L}(\pi) \neq \emptyset$. The logical formulas are defined using the following BNF description:

$$
\varphi::=p|\neg \varphi| \varphi \wedge \psi\left|K_{i} \varphi\right|[\pi] \varphi
$$

where $p \in P, i \in I$ and $\pi \in \mathcal{L}_{\text {obs }}$.

An important result from this paper is the bisimilarity; a binary relation $R$ between two epistemic expectations models $\mathcal{M}=(S, \sim, V, \operatorname{Exp})$ and $\mathcal{N}=$ $\left(S^{\prime}, \sim^{\prime}, V^{\prime}, E x p^{\prime}\right)$ is called bisimilarity if for every $s \in S$ and $s^{\prime} \in S^{\prime}$, if we have $\left(s, s^{\prime}\right) \in R$, then:

$$
\begin{aligned}
& \text { Propositional invariance: } V(s)=V^{\prime}\left(s^{\prime}\right) \\
& \text { Observation invariance: } \mathcal{L}(\operatorname{Exp}(s))=\mathcal{L}\left(\operatorname{Exp}\left(s^{\prime}\right)\right) \\
& \text { Zig: } s \sim_{i} t \in \mathcal{M} \Longrightarrow \text { exists } t^{\prime} \in \mathcal{N} \\
& \text { Such that } s^{\prime} \sim_{i}^{\prime} t^{\prime} \text { and } t R t^{\prime} \\
& \text { Zag: } s^{\prime} \sim_{i}^{\prime} t^{\prime} \in \mathcal{N} \Longrightarrow \text { exists } t \in \mathcal{M} \\
& \text { such that } s \sim_{i} t \text { and } t R t^{\prime}
\end{aligned}
$$

The article also introduce the bisimilarity invariance: for two epistemic states $\mathcal{M}, s$ and $\mathcal{N}, s^{\prime}$, the following two statements are equivalent:

i) $\mathcal{M}, s \leftrightarrow \mathcal{N}, s^{\prime}$

ii) for all $\varphi: \mathcal{M}, s \models \varphi \Longleftrightarrow \mathcal{N}, s^{\prime} \models \varphi$ 
Updated models. Let $w$ be an observation over $\Sigma$, and $\mathcal{M}=(S, \sim, V, \operatorname{Exp})$ an epistemic expectation model. The, the updated model is denoted with $\left.\mathcal{M}\right|_{w}=\left(S^{\prime}, \sim^{\prime}, V^{\prime}, E x p^{\prime}\right)$, where $S^{\prime}=\{s \mid \mathcal{L}(\operatorname{Exp}(s)-w) \neq \emptyset\}, \sim_{i}^{\prime}=\left.\sim_{i}\right|_{S^{\prime} \times I \times S^{\prime}}$, $V^{\prime}=\left.V\right|_{S^{\prime}}$ and $\operatorname{Exp}^{\prime}(s)=\operatorname{Exp}(s)-w$, where $\pi-w=\{v \mid w v \in \mathcal{L}(\pi)\}$.

Temporal models. Let $\mathcal{M}=(S, \sim, V, \operatorname{Exp})$ be an epistemic expectation model. Then the temporal model is called $E T(\mathcal{M})$ and is defined as $E T(\mathcal{M})=$ $\left(H, \rightarrow_{a}, \sim_{i}^{\prime}, V^{\prime}\right)$, where $H=\{(s, w) \mid s \in S, w=\varepsilon$ or $w \in \mathcal{L}(\operatorname{Exp}(s))\},(s, w) \rightarrow_{a}$ $(t, v) \Longleftrightarrow s=t$ and $v=w a, a \in \Sigma,(s, w) \sim_{i}(t, v) \Longleftrightarrow s \sim_{i} t$ and $w=v$ and $p \in V^{\prime}(s, w) \Longleftrightarrow p \in V(s)$.

Using temporal models, is it proved in this paper that $\mathcal{M}, s \models \varphi \Longleftrightarrow$ $\operatorname{ET}(\mathcal{M}),(s, \varepsilon) \models_{E P D L} \varphi$, so the system is complete by the completeness of dynamic epistemic logic.

\section{DELP - Dynamic Epistemic Logic for Protocols}

In order to define our system, we firstly recall the dynamic epistemic logic [9]. Dynamic epistemic logic is a dynamic logic [6] to which is added the knowledge operator $K$ from epistemic logic. There are two sets, $\Pi$ - the set of programs, and $\Phi$ - the set of formulas, with $\Pi_{0}$ - set of atomic programs, and $\Phi_{0}$ - set of atomic formulas. The language is described using the following BNF:

$$
\varphi::=p|\neg \varphi| \varphi \rightarrow \varphi\left|K_{i} \varphi\right|[\alpha] \varphi
$$

where $p \in \Phi_{0}, \varphi \in \Phi, i$ is an arbitrary agent and $\alpha \in \Pi$.

The evaluation models are Kripke models $\mathcal{M}=(R, \sim, V)$, where $R$ is the finite set of accessible worlds, $\sim$ is the accessibility relationship between worlds, and $V$ is the evaluation from dynamic logic: for a formula $\varphi \in \Phi, V(\varphi) \subseteq R$, and for a program $\alpha \in \Pi, V(\pi) \subseteq R \times R$.

Interpretation of formulas in this models are inductively defined as:

$$
\begin{gathered}
\mathcal{M}, s \models p \Longleftrightarrow v \in V(s) \\
\mathcal{M}, s \models \varphi \wedge \psi \Longleftrightarrow \mathcal{M}, s \models \varphi \text { and } \mathcal{M}, s \models \psi \\
\mathcal{M}, s \models \neg \varphi \Longleftrightarrow \mathcal{M}, s \not \varphi \\
\mathcal{M}, s \models K_{i} \varphi \Longleftrightarrow \text { for all } t \text { such that } s \sim_{i} t, \\
\text { we have } \mathcal{M}, t \models \varphi \\
\mathcal{M}, s \models[\alpha] \varphi \Longleftrightarrow \text { for all } t \in R \text { such that } \\
\quad(s, t) \in V(\alpha), \text { we have } \mathcal{M}, t \models \varphi
\end{gathered}
$$

We also have the following operators for programs:

$$
\begin{aligned}
V\left(\alpha_{1} \cup \alpha_{2}\right) & =V\left(\alpha_{1}\right) \cup V\left(\alpha_{2}\right) \\
V\left(\alpha_{1} ; \alpha_{2}\right) & =V\left(\alpha_{1}\right) \circ V\left(\alpha_{2}\right) \\
V\left(\alpha^{*}\right) & =\bigcup_{n \geq 0} V(\alpha)^{n}
\end{aligned}
$$

The deductive system contains all instances of propositional tautologies to 
which are added the following axioms:

$$
\begin{aligned}
& K_{a}(\varphi \rightarrow \psi) \rightarrow\left(K_{a} \varphi \rightarrow K_{a} \psi\right) \\
& K_{a} \varphi \rightarrow \varphi \\
& K_{a} \varphi \rightarrow K_{a} K_{a} \varphi \\
& \neg K_{a} \varphi \rightarrow K_{a} \neg K_{a} \varphi \\
& {[\alpha](\varphi \rightarrow \psi) \rightarrow([\alpha] \varphi \rightarrow[\alpha] \psi)} \\
& {[\alpha](\varphi \wedge \psi) \leftrightarrow[\alpha] \varphi \wedge[\alpha] \psi} \\
& {[\alpha \cup \beta] \varphi \leftrightarrow[\alpha] \varphi \wedge[\alpha] \psi} \\
& {[\alpha ; \beta] \varphi \leftrightarrow[\alpha][\beta] \varphi}
\end{aligned}
$$

Deductive rules are modus ponens, generalization from dynamic logic and necessity from epistemic logic:

$$
(M P) \frac{\varphi \varphi \rightarrow \psi}{\psi} ;(G E N) \frac{\varphi}{[\alpha] \varphi} ;(N E C) \frac{\varphi}{K_{i} \varphi}
$$

This system is known as the $P A$-system in [9], and it is proved sound and complete [9, p. 187-188].

\subsection{DELP}

In this subsection we define $D E L P$, a logic based on dynamic epistemic logic, enriched with a set of actions collected during the execution of the protocol and a grammar for messages, together with a system of deduction for knowledge based on actions.

\subsubsection{Syntax}

Let Agent be the set of agents and let Func be a set of (encryption) functions. We consider the sets $\Phi$ and $\Pi$ like in dynamic epistemic logic, with $\Phi_{0}$ the set of atomic formulas, and $\Pi_{0}$ defined by

$$
\Pi_{0}:=\left.\left\{\operatorname{send}_{i}, \operatorname{recv}_{i}\right\}\right|_{i \in \text { Agent }}
$$

The elements of $\Pi_{0}$ are protocols actions: we read send $d_{i}$ as "the agent $i$ sends" and we read recv $_{i}$ as "the agent $i$ receives".

In the following we define messages and formulas. In a security protocol, a message contains clear texts, keys, nonces, and agents identities. The possible operations are messages concatenation and messages encryption. Following [7], the grammar for messages is:

$$
\begin{gathered}
m::=\operatorname{text}(m)\left|\operatorname{key}_{m}(i, j)\right| \operatorname{nonce}(m) \mid \operatorname{agent}(i) \\
|(m, m)|\{m\}_{m} \mid f(m, \ldots, m)
\end{gathered}
$$

where $i, j \in$ Agent and $f \in$ Func. In the sequel we will use $t$ for texts, $k$ for keys, $n$ for nonces and $i, j$ for agents. Based on [7], we define the following 
deductive system on messages:

$$
\begin{aligned}
& \frac{}{\text { nonce }(m)} \frac{k e y_{k}(i, j)}{k e y_{k}(j, i)} \frac{m_{1} \quad m_{2}}{\left(m_{1}, m_{2}\right)} \\
& \frac{t \quad k}{\{t\}_{k}} \quad \frac{\{t\}_{k}}{t} \quad \frac{t_{1}, t_{2}, \ldots, t_{n}}{f\left(t_{1}, t_{2}, \ldots, t_{n}\right)}
\end{aligned}
$$

Finally, we are able to define the $D E L P$ formulas:

$$
\varphi::=p|\neg \varphi| \varphi \rightarrow \varphi\left|K_{i} \varphi\right|[\alpha] \varphi \mid @ \mu
$$

Note that our formulas are the usual formulas of dynamic epistemic logic with protocol actions instead of programs, endowed with the @-operator which converts a message into a formula.

\subsubsection{Semantics}

The models that we use are Kripke models like in dynamic epistemic logic, $\mathcal{M}=(R, \sim, V)$ which we extend with $\operatorname{Exp}$ set, a knowledge set with information collected from protocol runs.

Definition 1. Let $\mathcal{M}=(R, \sim, V, E x p)$ be a DELP model, where

1. $R$ is the finite set of accessible worlds;

2. $\sim:=\bigcup_{i \in \text { Agent }} \sim_{i}$ represents the accessibility relationship between worlds, based on epistemic relation;

3. $V$ is the evaluation function from dynamic logic: $V(\varphi) \subseteq R$ for any $\varphi \in \Phi$, and $V(\alpha) \subseteq R \times R$, for any $\alpha \in \Pi$;

4. Exp is the knowledge set: for any $s \in R, \operatorname{Exp}(s)$ represents the set of all knowledge inferred up to s-th round of the protocol;

5. for any agent $i, V\left(\right.$ send $\left._{i}\right) \subseteq \sim_{i}$ and $V\left(\operatorname{recv}_{i}\right) \subseteq \sim_{i}$.

Having this models, we can interpret @ $\mu$ formula as:

$$
\mathcal{M}, s \models @ \Longleftrightarrow \mu \in \operatorname{Exp}(s)
$$

The other formulas have the interpretation from the dynamic epistemic logic:

$$
\begin{aligned}
& \mathcal{M}, s \models p \Longleftrightarrow v \in V(s) \\
& \mathcal{M}, s \models \varphi \wedge \psi \Longleftrightarrow \mathcal{M}, s \models \varphi \text { and } \mathcal{M}, s \models \psi \\
& \mathcal{M}, s \models \neg \varphi \Longleftrightarrow \mathcal{M}, s \not \models \varphi \\
& \mathcal{M}, s \models K_{i} \varphi \Longleftrightarrow \text { for all } t \text { such that } s \sim_{i} t, \\
& \quad \text { we have } \mathcal{M}, t \models \varphi \\
& \mathcal{M}, s \models[\alpha] \varphi \Longleftrightarrow \text { for all } t \in R \text { such that } \\
& \quad(s, t) \in V(\alpha), \text { we have } \mathcal{M}, t \models \varphi
\end{aligned}
$$




\subsubsection{Deductive system}

The deductive system contains all instances of propositional tautologies to which are added the following axioms from dynamic epistemic logic:

$$
\begin{aligned}
& K_{a}(\varphi \rightarrow \psi) \rightarrow\left(K_{a} \varphi \rightarrow K_{a} \psi\right) \\
& K_{a} \varphi \rightarrow \varphi \\
& K_{a} \varphi \rightarrow K_{a} K_{a} \varphi \\
& \neg K_{a} \varphi \rightarrow K_{a} \neg K_{a} \varphi \\
& {[\alpha](\varphi \rightarrow \psi) \rightarrow[[\alpha] \varphi \rightarrow[\alpha] \psi)} \\
& {[\alpha](\varphi \wedge \psi) \leftrightarrow[\alpha] \varphi \wedge[\alpha] \psi} \\
& {[\alpha \cup \beta] \varphi \leftrightarrow[\alpha] \varphi \wedge[\alpha] \psi} \\
& {[\alpha ; \beta] \varphi \leftrightarrow[\alpha][\beta] \varphi}
\end{aligned}
$$

In addition, we have the following specific axiom, that is necessary to have a correspondence between states; if the agent $i$ performs an action within the protocols (sends or receives a message), then he knows the message:

$$
\left[\operatorname{send}_{i}\right] @ m \vee\left[\operatorname{recv}_{i}\right] @ m \rightarrow K_{i} @ m
$$

The soundness of this system is given by the soundness of the dynamic epistemic logic [9, p. 187-188], and all that remains for us to prove is the soundness of the specific axiom.

Lemma 1. Axiom $\left[\right.$ send $\left._{i}\right] @ m \vee\left[\right.$ recv $\left._{i}\right] @ m \rightarrow K_{i} @ m$ is sound.

Proof. Let $\mathcal{M}=(R, \sim, V, E x p)$ be a $D E L P$ model and $s \in R$ an arbitrary state.

$$
\begin{aligned}
& \mathcal{M}, s \models\left[\text { send }_{i}\right] @ m \Longleftrightarrow \text { for all } t \text { such that }(s, t) \in V\left(\text { send }_{i}\right), \\
& \quad \text { we have that } \mathcal{M}, t \models @ m
\end{aligned}
$$

but $V\left(\operatorname{send}_{i}\right) \subseteq \sim_{i}$, so

$$
\begin{aligned}
& \mathcal{M}, s \models\left[\operatorname{send}_{i}\right] @ m \Longleftrightarrow \text { for all } t \text { such that }(s, t) \in \sim_{i}, \\
& \quad \text { we have that } \mathcal{M}, t \models @ m \\
& \quad \Longleftrightarrow \mathcal{M}, s \models K_{i} @ m
\end{aligned}
$$

\subsubsection{Completeness}

In order to prove the completeness of $D E L P$, we follow ideas from $[8]$ and general results from dynamic epistemic logic.

Definition 2. [Restricted model] Let $\mu$ be a message and $\mathcal{M}=(R, \sim, V$, Exp $)$ a DELP model. Then, the restricted model is defined as

$$
\left.M\right|_{\mu}=\left(R^{\prime}, \sim^{\prime}, V^{\prime}, \operatorname{Exp}^{\prime}\right)
$$

where $R^{\prime}=\{s \mid \operatorname{Exp}(s)-\mu \neq \emptyset\}, \sim_{i}^{\prime}=\left.\sim_{i}\right|_{R^{\prime} \times R^{\prime}}, V^{\prime}=\left.V\right|_{R^{\prime}}$, and $\operatorname{Exp}^{\prime}(s)=$ $\operatorname{Exp}(s)-\mu$. 
Definition 3. [Temporal model] Let $\mathcal{M}=(R, \sim, V$, Exp $)$ be a DELP model. We define

$$
E T(\mathcal{M})=\left(H, \rightarrow, \sim^{\prime}, V^{\prime}\right)
$$

where

- $H=\{(s, m) \mid s \in R, m \in \operatorname{Exp}(s)\}$;

- $(s, m) \rightarrow\left(s^{\prime}, m^{\prime}\right)$ if and only if $s=s^{\prime}$ and $\{m\} \vdash m^{\prime}$ using the deduction system (37);

- $(s, m) \sim^{\prime}\left(s^{\prime}, m^{\prime}\right)$ if and only if $s \sim s^{\prime}$ and $m \equiv m^{\prime}$ where $\equiv$ is the logic equivalence;

- $p \in V^{\prime}(s, m)$ if and only if $p \in V(s)$

Having $\mathcal{N}=E T(\mathcal{M})$ a temporal model, we inductively define the following interpretation of formulas:

$$
\begin{aligned}
& \mathcal{N}, w \models p \Longleftrightarrow p \in V(w) \\
& \mathcal{N}, w \models \neg \varphi \Longleftrightarrow \mathcal{N}, w \not \models \varphi \\
& \mathcal{N}, w \models \varphi \wedge \psi \Longleftrightarrow \mathcal{N}, w \models \varphi \text { and } \mathcal{N}, w \models \psi \\
& \mathcal{N}, w \models K_{i} \varphi \Longleftrightarrow \text { for all } v \in \mathcal{N}, \text { if } w \sim_{i} v, \\
& \quad \text { then } \mathcal{N}, v \models \varphi \\
& \mathcal{N}, w \models[\alpha] \varphi \Longleftrightarrow \text { for all } \mu \in \operatorname{Exp}(\alpha), \\
& w \rightarrow v \text { implies } \mathcal{N}, w \models \varphi
\end{aligned}
$$

Definition 4. [Bisimilarity] Based on [8, Def. 11], we have that the binary relation $\rho \subseteq \mathcal{M} \times \mathcal{N}$, for two DELP models $\mathcal{M}=(R, \sim, V$, Exp $)$ and $\mathcal{N}=$ $\left(R^{\prime}, \sim^{\prime}, V^{\prime}, E x p^{\prime}\right)$ is called bisimilarity if for any $v \in R$ and $v^{\prime} \in R^{\prime}$, if we have $v \rho v^{\prime}$, then:

$$
\begin{array}{r}
\text { Propositional invariance } \\
V(v)=V^{\prime}\left(v^{\prime}\right)
\end{array}
$$

\section{Observation invariance}

$$
\begin{array}{r}
\text { Zig } v \sim_{i} w \in \mathcal{M} \Longrightarrow \text { exists } w^{\prime} \in \mathcal{N} \\
\text { such that } v^{\prime} \sim_{i}^{\prime} w^{\prime} \text { and } w \rho w^{\prime} \\
\text { Zag } v^{\prime} \sim_{i}^{\prime} w^{\prime} \in \mathcal{N} \Longrightarrow \text { exists } w \in \mathcal{M} \\
\text { such that } v \sim_{i} w \text { and } w \rho w^{\prime}
\end{array}
$$

Theorem 1. [Bisimilarity invariance] For two DELP states $\mathcal{M}, v$ and $\mathcal{N}, v^{\prime}$, the following two statements are equivalent:

$$
\begin{aligned}
& \text { (i) } \mathcal{M}, v \leftrightarrow \mathcal{N}, v^{\prime} \\
& \text { (ii) for all } \varphi: \mathcal{M}, v \models \varphi \Longleftrightarrow \mathcal{N}, v^{\prime} \models \varphi
\end{aligned}
$$

The proof is the same as [8, Prop. 12]. 
Theorem 2. [Completeness] Let $\mathcal{M}=(R, \sim, V$, Exp $)$ be a DELP model, $\varepsilon$ the initial knowledge and $\varphi \in \Phi$ a formula. Then

$$
\mathcal{M}, v \models \varphi \Longleftrightarrow E T(\mathcal{M}),(s, \varepsilon) \models \varphi
$$

Proof. We follow the proof from [8, Prop. 14]. The booleean and epistemic cases are immediate from the temporal model construction. For $\varphi:=[\alpha] \psi$ we assume that $\mathcal{M}, v \models[\alpha] \psi$, but $\operatorname{ET}(\mathcal{M}),(v, \epsilon) \not \models[\alpha] \psi$. Then, exists $m \in \operatorname{Exp}(v)$ such that $E T(\mathcal{M}),(v, m) \not \forall \psi$. From the construction of $E T(\mathcal{M})$, the definition of worlds is $H=\{(s, m) \mid s \in R, m \in \operatorname{Exp}(s)\}$, so $m \in \operatorname{Exp}(v)$. But $m$ is a message, then exists the restricted model $\left.\mathcal{M}\right|_{m}$. From bisimilarity, we have that $E T\left(\left.\mathcal{M}\right|_{m}\right),(v, \epsilon)$ is bisimilar with $E T(\mathcal{M}),(v, m)$. Then $E T\left(\left.\mathcal{M}\right|_{m}\right),(v, \varepsilon) \models$ $\neg \psi$. From the induction hypothesis, we have $\mathcal{M}, v \models \neg \psi$, which contradicts $\mathcal{M}, v \models[\alpha] \psi$.

We have that the DELP system is complete.

\section{Implementation in Lean}

In this section we will present the implementation of our system in Lean [1] prover assistant based on [2], and then we will prove the corectness of $B A N$ deduction rules in DELP.

\subsection{Language}

To implement $D E L P$, we have the following inductive types:

1. For messages:

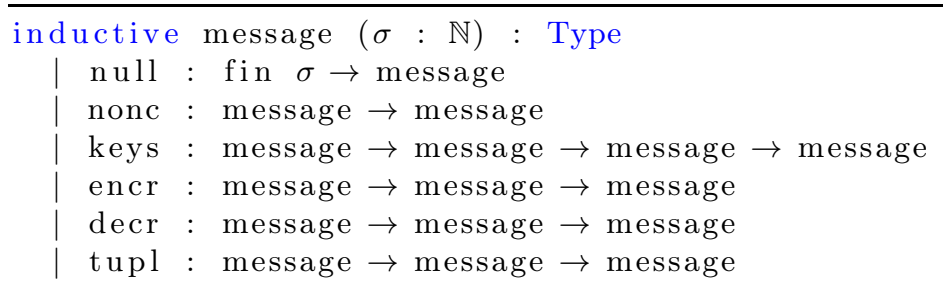

2. For programs:

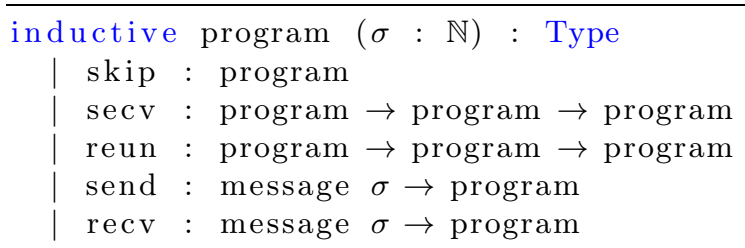

3. For formulas:

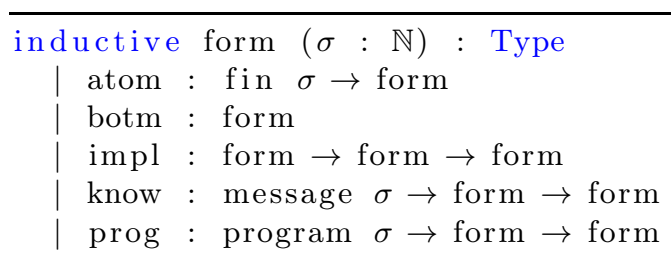




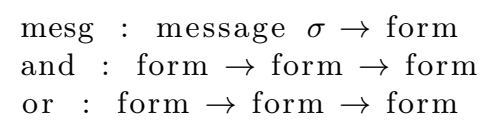

We make the following notations:

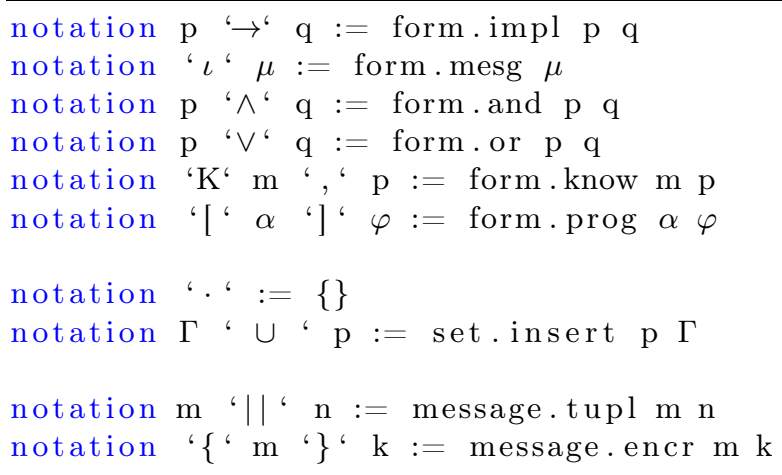

\subsection{Deductive system}

In order to be able to check security properties using DELP, we have two add two deduction hypotheses that help us specify symmetric key protocols:

$$
\begin{aligned}
& @\{m\}_{k} \wedge @ k e y_{k}(i, j) \rightarrow\left[\operatorname{send}_{i}\right] @ m \vee\left[\operatorname{send}_{j}\right] @ m \\
& @ k e y_{k}(i, j) \rightarrow K_{i} @ k \vee K_{j} @ k
\end{aligned}
$$

Observation 1. The first deduction hypothesis of the system represents a rule of honesty of the participating agents; its need is highlighted in the modeling of the BAN logic: if there is an encrypted message with the communication key $k$, and the communication key $k$ is a key known to the agents $i$ and $j$, then the message is transmitted by only one of them.

Observation 2. The second deduction hypothesis is a rule for modeling symmetric key protocols: if the $k$ key is a communication key between $i$ and $j$, then each of them knows it.

We define the following context, a set $\Gamma$ of statements:

def $\operatorname{ctx}(\sigma: \mathbb{N}):$ Type $:=\operatorname{set}($ form $\sigma)$

The deductive system is:

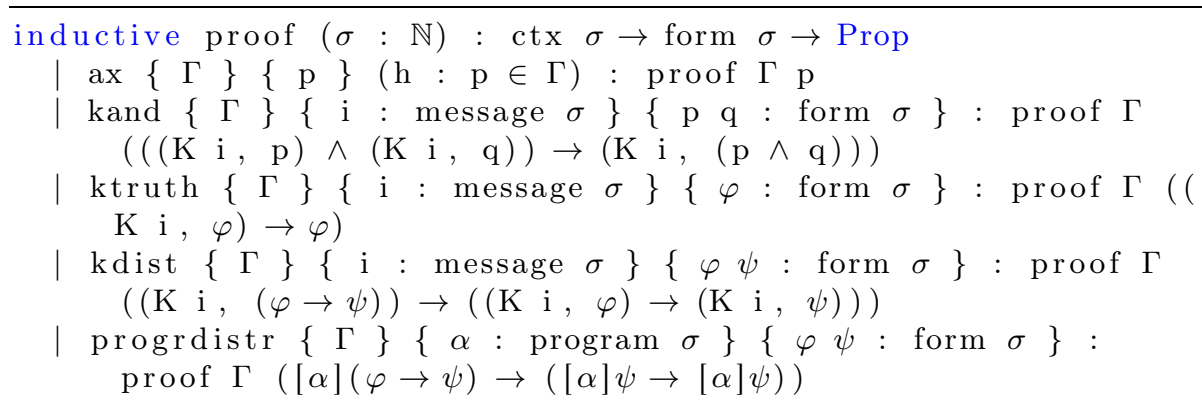




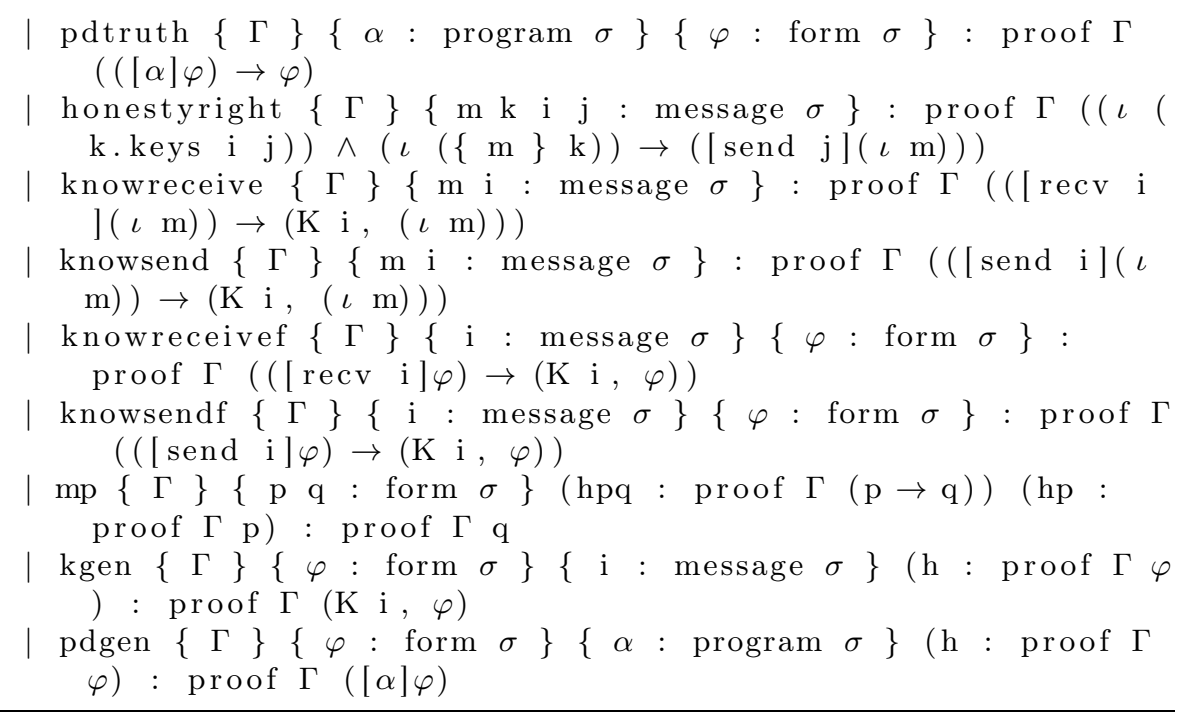

\subsection{BAN Rules Verification}

In order to be able to verify the corectness of the BAN rules, we translate them our logic. We use the following correspondence:

1. formula $i \mid \equiv m$ is translated as $K_{i} @ m$ and it means $i$ knows $m$ in current state;

2. formula $i \triangleleft m$ means that $i$ receives $m$ and is translated as $\left[r e c v_{i}\right] @ m$;

3. formula $i \mid \sim m$ is translated as $\left[\right.$ send $\left._{i}\right] @ m$;

4. formula $i \Rightarrow m$ means that $i$ has jurisdiction over $m$, so the agent knows $m$ and $m$ is true: $K_{i} @ m \rightarrow @ m$;

5. formula $i \stackrel{k}{\leftrightarrow} j$ is translated as @keyk $(i, j)$;

6. formula \# $(m)$ is translated as @nonce $(m)$.

Now, we can prove that the translations in DELP of the most important BAN inference rules (according to [5]) are sound. In the sequel, using Lean, we give the proofs only for the Message Meaning rule and for the Jurisdiction rule, few other rules are analysed in the Appendix.

Lemma 2. The Message Meaning rule for shared key is a correct rule in the DELP system.

$$
\frac{i \mid \equiv j \stackrel{k}{\leftrightarrow} i \quad i \triangleleft\{m\}_{k}}{i|\equiv j| \sim m}
$$

Proof. We will prove this using Lean.

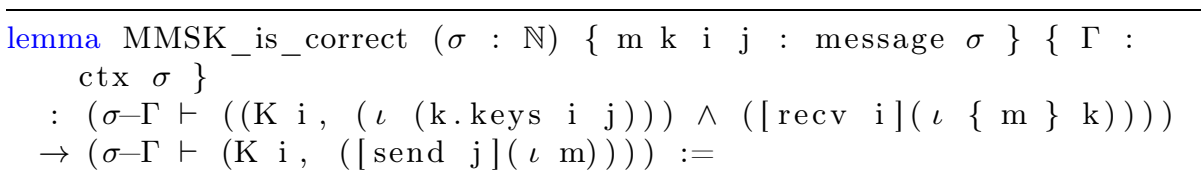


$\lambda \mathrm{h}$, kgen

$\$$ mp honestyright

$\$$ mp ktruth

$\$ \mathrm{mp}$ kand

$\$$ andintro

( andleft $\mathrm{h}$ )

(mp knowreceive $\$$ andright $\mathrm{h}$ ).

A much easier demonstration is for the jurisdiction rule, because it uses the $K$ operator distributivity over implication:

Lemma 3. Jurisdiction rule is a correct rule in DELP system.

$$
\frac{i|\equiv j \Rightarrow m \quad i| \equiv j \mid \equiv m}{i \mid \equiv m}
$$

Proof. We will prove this using Lean.

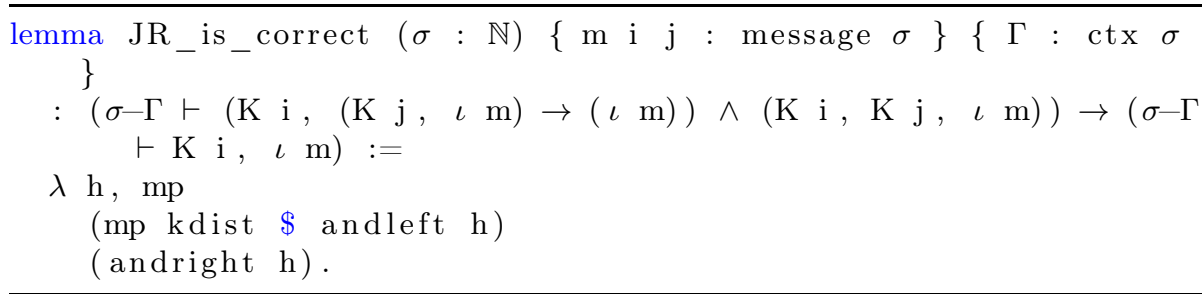

\section{$5 \quad$ Needham-Schroeder protocol implementation in Lean}

In this section we will analyze the Needham-Schroeder protocol and we will implement the specification in Lean, in order to prove some security properties. We recall the exchange of messages in Needham-Schroeder protocol:

$$
\begin{aligned}
& A \rightarrow S: A, B, N_{a} \\
& S \rightarrow A:\left\{N_{a}, B, K_{a b},\left\{K_{a b}, A\right\}_{K_{b s}}\right\}_{K_{a s}} \\
& A \rightarrow B:\left\{K_{a b}, A\right\}_{K_{b s}} \\
& B \rightarrow A:\left\{N_{b}\right\}_{K_{a b}} \\
& A \rightarrow B:\left\{N_{b}-1\right\}_{K_{a b}}
\end{aligned}
$$

\subsection{Protocol description in Lean}

In this subsection we will formalize the specification in $D E L P$ and then we will implement every $D E L P$ formula in Lean.

First step: intialization 
The initial knowledge of agents are:

$$
\begin{aligned}
& K_{A}\left(@ N_{A} \wedge @ k e y_{K_{A S}}(A, S)\right) \\
& K_{S}\left(@ k e y_{K_{A S}}(A, S) \wedge @ k e y_{K_{B S}}(B, S) \wedge @ k e y_{K_{A B}}(A, B)\right) \\
& K_{B} @ k e y_{K_{B S}}(B, S)
\end{aligned}
$$

In Lean we have:

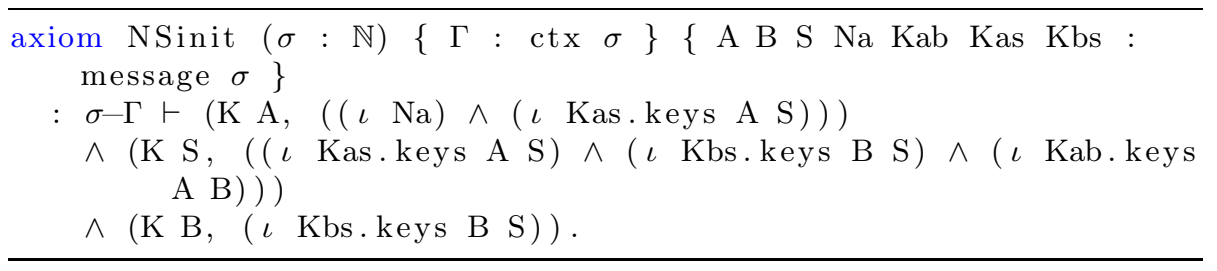

First round: exchange of messages between $A$ and $S$

In $D E L P$ we have:

$$
\left[\operatorname{send}_{A}\right]\left[\operatorname{recv}_{S}\right] @ N_{A}
$$

with the corresponding Lean implementation:

axiom $\operatorname{NS} 1 \operatorname{AtoS}(\sigma: \mathbb{N})\{\Gamma: \operatorname{ctx} \sigma\}\{$ A S Na $:$ message $\sigma\}$
$\quad: \sigma-\Gamma \vdash[$ send A] [recv S] $(\iota \mathrm{Na})$.

Second round: exchange of messages between $S$ and $A$

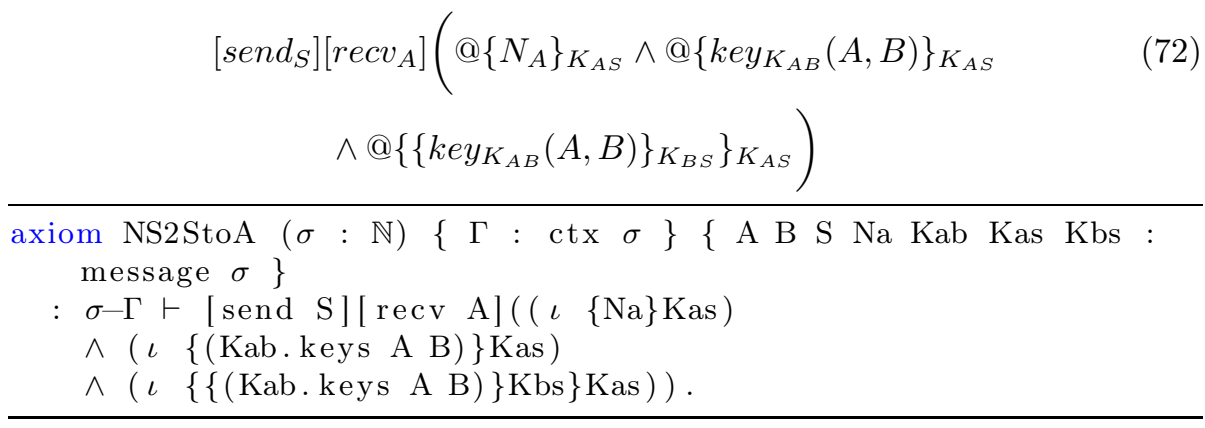

\section{Third round: exchange of messages between $A$ and $B$}

This is the last round we can formalize using DELP system at the moment. For the next two round, we need a more expressive system, that can model both the knowledge and belief. However, up to this point we can prove that $K_{a b}$ is a common secret between $A$ and $B$, but we cannot prove the mutual authentication of these two agents.

$$
\left[\operatorname{send}_{A}\right]\left[\operatorname{recv}_{B}\right] @\left\{\operatorname{key}_{K_{A B}}(A, B)\right\}_{K_{B S}}
$$

axiom NS3AtoB $(\sigma: \mathbb{N})\{\Gamma: \operatorname{ctx} \sigma\}\{$ A B S Kab Kbs : message

$$
\sigma\}
$$

: $\sigma-\Gamma \vdash[$ send A] [recv B] $\iota\{($ Kab.keys A B $)\}$ Kbs. 


\subsection{Verifying security properties of Needham-Schroeder}

In order to prove some security properties, we must prove the following lemma that we will use further.

Lemma 4. Let $\Gamma$ be a set of statements, $i$ and $j$ two agents and $\varphi$ a formula. Then $\Gamma \vdash\left[\right.$ send $\left._{i}\right]\left[\right.$ recv $\left._{j}\right] \varphi$ implies $\Gamma \vdash K_{j} \varphi$.

Proof. We will prove this lemma using Lean.

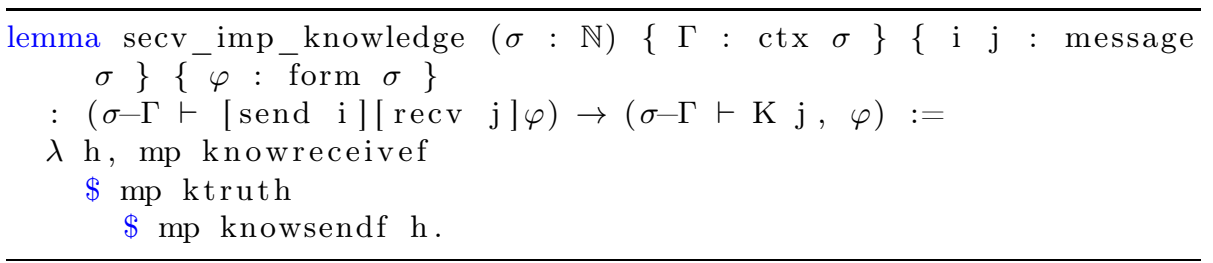

We can prove that the agent $A$ knows the communication key between $A$ and $B$.

Theorem 3. In Needham-Schroeder protocol, the agent A knows the communication key between $A$ and $B$.

Proof. We will prove this theorem using Lean.

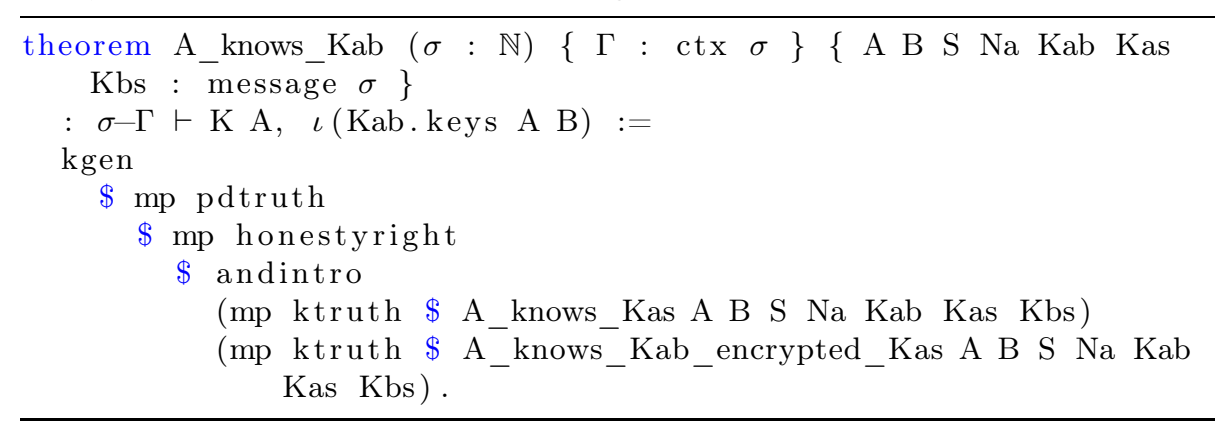

In a similar way, we can prove that also $B$ knows the communication key between $A$ and $B$.

Theorem 4. In Needham-Schroeder protocols, the agent B knows the communication key between $A$ and $B$.

Proof. We will prove this theorem using Lean.

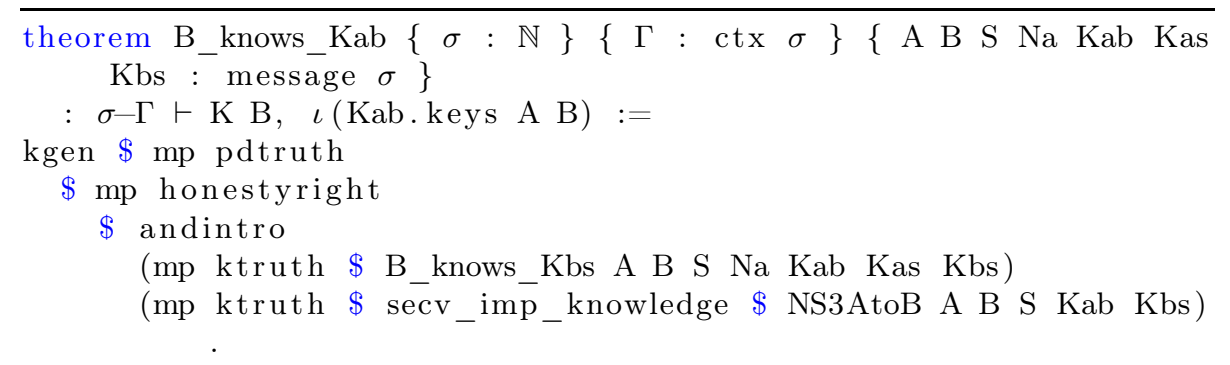


We have now that $K_{a b}$ is a common secret between $A$ and $B$, but we cannot prove that we also have a mutual authentication. We know that $K_{A} @ k e y_{K_{a b}}(A, B) \wedge$ $K_{B} @ k e y_{K_{a b}}(A, B)$, but we don't know if $K_{A} K_{B} @ k e y_{K_{a b}}(A, B)$ and $K_{B} K_{A} @ k e y_{K_{a b}}(A, B)$.

\section{Conclusion and further work}

The system DELP is closely related to the system POL (Public observation logic [5]), but it has a different semantics for $[\alpha] \varphi$ : the updated models of $P O L$ are replaced by $D E L$ models [9], while the set Exp represents the "adversary knowledge" (defined as in the operational semantics from [7]) and not the "expected observations" (as in $P O L$ ). Even if our system is simpler than the one from [5], we are able to translate $B A N$ logic and to validate $B A N$ inference rules.

Our work so far shows that DELP is a good candidate for modelling and analysing security protocols. We are aimig to define a system that has a rigourous theoretical development: it is complete and all proofs are certified by Lean implementations.

At this stage we've already noticed that further refinements are needed: so far we used "knowledge" operators but, in order to increse our system expressiveness, we would like to model the epistemic "trust"; we also consider adding a temporal behaviour, in order to be able to model the property of freshness since, currently, we use a weaker variant, namely the uniqueness on the system (nonce). Last but not least, we consider adding the probabilistic interpretation, following the initial idea from [5].

On the implementation side in Lean, we will add the proof for the completeness theorem and we will keep all the theoretical results automatically verified for any subsequent modification.

\section{References}

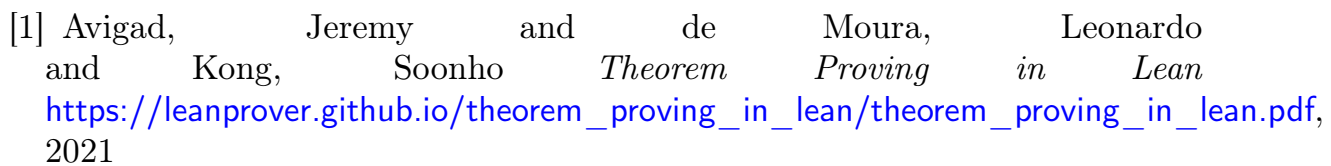

[2] Bentzen, Bruno. "A Henkin-style completeness proof for the modal logic S5." arXiv preprint arXiv:1910.01697 (2019).

[3] Blackburn, Patrick, Maarten De Rijke, and Yde Venema. Modal logic: graph. Darst. Vol. 53. Cambridge University Press, 2002.

[4] Burrows, Michael, Martin Abadi, and Roger Michael Needham. "A logic of authentication." Proceedings of the Royal Society of London. A. Mathematical and Physical Sciences 426.1871 (1989): 233-271.

[5] Halpern, Joseph Y., Ron van der Meyden, and Riccardo Pucella. "An epistemic foundation for authentication logics." arXiv preprint arXiv:1707.08750 (2017). 
[6] Harel, David, Dexter Kozen, and Jerzy Tiuryn. "Dynamic logic." Handbook of philosophical logic. Springer, Dordrecht, 2001. 99-217.

[7] Cremers, Cas, and Sjouke Mauw. "Operational semantics." Operational Semantics and Verification of Security Protocols. Springer, Berlin, Heidelberg, 2012. 13-35.

[8] Van Ditmarsch, Hans, et al. "Hidden protocols: Modifying our expectations in an evolving world." Artificial Intelligence 208 (2014): 18-40.

[9] Van Ditmarsch, Hans, Wiebe van Der Hoek, and Barteld Kooi. Dynamic epistemic logic. Vol. 337. Springer Science \& Business Media, 2007. 